\title{
MEASUREMENT OF SCI PATIENT'S BUTTOCK PRESSURE ON WHEELCHAIR AND BED
}

\author{
Yoshio Tanimoto ${ }^{1)}$, Hirosuke Takechi ${ }^{1)}$, Akihiro Tokuhiro ${ }^{1)}$, Hideo Takechi ${ }^{1)}$ \\ and Hideki Yamamoto ${ }^{2)}$
}

1) Kibikogen Rehabilitation Center, 7511 Yoshikawa, Jobogun-Kayocho, Okayama, Japan,

2) Okayama University, Faculty of Education, 3-1-1 Tushimanaka Okayama, Japan

\begin{abstract}
The prevention of the pressure sore is a serious problem for patients with spinal cord injuries (SCI). It is the most important for SCI patients to relieve the buttock pressure for prevention of pressure sore. Many kinds of pressure-relieving cushions and mattress have been developed to distribute the weight evenly and widely. We previously measured the buttock pressure of SCI patients using Tekscan pressure measurement system to evaluate the pressure distribution. In this study, we propose the evaluating system of the buttock pressure distribution. Using this system, the medical staffs can easily adjust the air quantity of the air cushion for each SCI patient for prevention of the pressure sores. Moreover we measured the buttock pressure in four kinds of posture on the bed to indicate the pressure relieving effect of the mattress. The air mattress has a high pressure relieving effect at the sacro-coccygeal regions in all postures on the bed.
\end{abstract}

Keywords: - Pressure measurement, Pressure distribution, Pressure sore, Spinal cord injury, Wheelchair cushion

\section{INT RODUCTION}

The pressure and tolerance of tissue are big factors in the development of pressure sores[1]. Many kinds of pressure-relieving cushions and mattresses have been developed to distribute weight evenly, and over the widest surface area of the body [2], [3]. In rehabilitation medicine, physicians and physical therapists (PTs) assist the patients in selecting wheelchair cushions and mattresses. They sometimes adjust the cushion, if necessary. It is very important for physicians and PTs to evaluate the pressure distribution of the cushion and mattress selected.

D.Brienza et al suggested that it is not pressure alone but rather the resulting tissue deformation that results in the development of pressure sores [4]. L. Conner reported on tissue deformation using a computed tomography (CT) scan [5]; however, these measurements do not adequately assist persons in rehabilitation medicine when selecting or adjusting wheelchair cushions and mattresses. Another approach, therefore, has been to measure the interface pressure using a pressure mapping system [6], [7]. We previously measured the buttock pressure distribution of SCI patients using the Tekscan pressure measurement system, and reported that the air cushion was the best among the five cushions tested, which consisted of the air cushion, a contour cushion, a gel cushion, the Cubicushion, and a urethane foam cushion [8]. We calculated six parameters to evaluate the buttock pressure distribution for SCI patients, and represented these parameters on a radar plot in order to help medical staff select and adjust wheelchair cushions [9].

In this study, we measured the buttock pressure of SCI patients, and proposed a system for evaluating buttock pressure on the wheelchair, in order to make the best air adjustment of the air cushion. Next, we measured the buttock pressure of SCI patients on beds, in order to assess the pressure relieving effect of the mattress, and the risk of pressure sore development when sitting up in bed.

\section{MEASUREMENT SYSTEM}

Figure 1 shows the Tekscan "Big-Mat" pressure measurement system. As a sensor seat consists of 48pixels x 43pixels, we can measure the buttock pressure at 2,064 points simultaneously with minimal error. This sensor seat is $2 \mathrm{~mm}$ thick and is soft, which allowed us to measure the pressure on soft objects, such as cushions and beds. The error caused by creep characteristics was large. After having the subjects sit on the wheelchair cushion for 1 minute, we measured the pressure in order to reduce the error caused by the creep characteristics of the sensor [8].

Figure 2 shows the 2-D representation of the pressure distribution. Data is displayed in 16 shades of gray. High-pressure areas are black, low are 


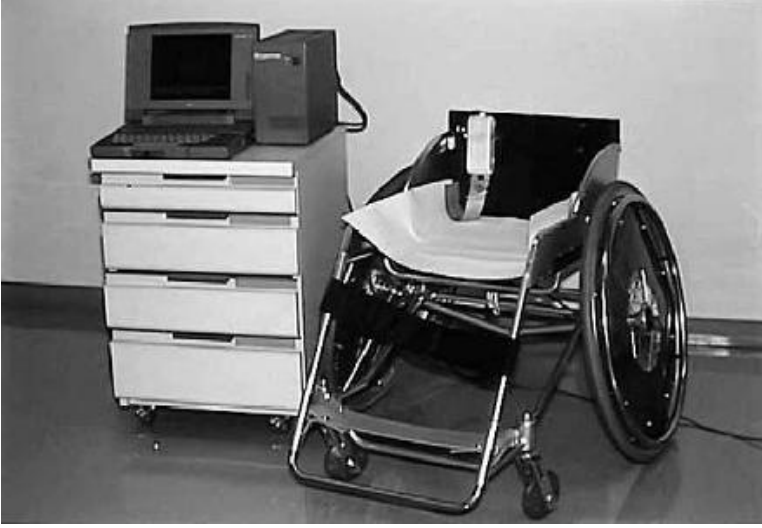

Fig.1 Tekscan pressure measurement system.
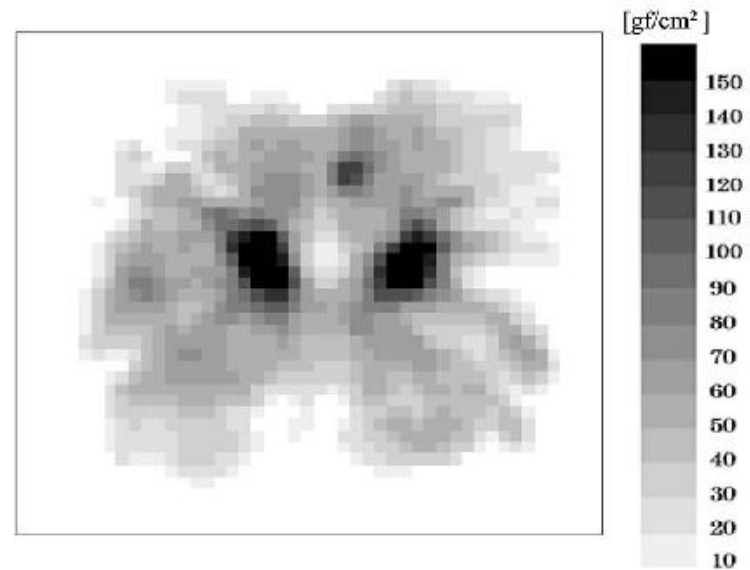

\section{Fig.2 2-D pressure distribution of SCI patient.}

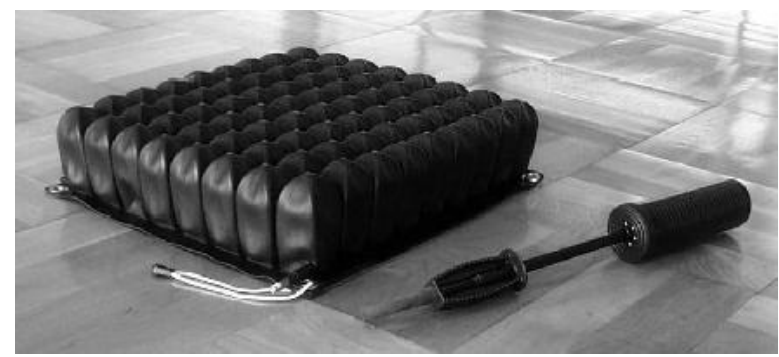

Fig.3 Air cushion.

white, and middle are gray. SCI patients tend to develop pressure sores in their ischial tuberosities, greater trochanter, and sacro- coccygeal regions. Areas of high pressure are observed at these areas for all subjects. In this SCI patient, the highpressure area is observed around both ischial tuberosities and at the sacro-coccygeal regions. The medical staff can learn the location of high-pressure area and its size from the 2-D representation of the pressure distribution. Moreover, he or she can find out, from this representation, whether the SCI patient anteriorly or posterioly sits on their wheelchair.

\section{MEASUREMENT ON WHEELCHAIR}

\subsection{WHEELCHAIR CUSHION}

The air cushion consisted of many rubber air cells, shown in Figure 3. SCI patients can easily adjust the quantity of air in the cells using an air pump. Most SCI patients use this cushion because of the even pressure distribution, and because of the ease of adjustment using the air pump. Because the influence of the air pressure on pressure distribution is large in the air cushion, it is important that the medical staff care fully adjust the air quantity in the cushion [10].

\subsection{EVALUATION SYSTEM}

It is necessary for medical personnel and their SCI patients to evaluate the pressure distribution in detail, using the parameters for adjustment of the air quantity in the air cushion. To assist medical personnel in evaluating the buttock pressure distribution of SCI patients, we made our calculations using the following six parameters to evaluate the pressure distribution, sitting balance, and patient posture on the wheelchair:

(1) Maximum pressure (M.P.)

(2) Contact area (C.A.)

(3) High-pressure area (H.A.)

(4) Tip rate (T.R.)

(5) Sitting balance (S.B.)

(6) Sitting position (S.P.)

The tip rate, sitting balance, and sitting position are calculated from the top-five projections. The horizontal top-five projection is calculated from the 2-D representation of the pressure distribution, as shown in Figure 4. The pixel values at the $\boldsymbol{i}$ - $\boldsymbol{t} \boldsymbol{h}$ horizontal line of an image, $f$, are rearranged so that they are in decreasing order. The top five pixel values, that is, the largest pixel value $\boldsymbol{g}(\boldsymbol{i}, \mathbf{1})$, the next-largest pixel value $g(i, 2)$, all the way down to the 5th-largest pixel value $\boldsymbol{g}(\boldsymbol{i}, 5)$, are taken from the sorted pixel values. The horizontal top-five projection at the $\boldsymbol{i}$-th line is then defined by

$$
\operatorname{TFPx}(i)=\frac{1}{5} \sum_{k=1}^{5} g(i, k) \text {. }
$$

The horizontal $\boldsymbol{T F P \boldsymbol { x }}$ is obtained by calculating $\boldsymbol{T F P x}(i)$ for each horizontal line. Figure 5 shows the horizontal $\boldsymbol{T F P \boldsymbol { x }}$, the vertical top-five projection (TFPy), and an approximate Gaussian curve around the peak point of the $\boldsymbol{T F P \boldsymbol { x }}$. The data $\boldsymbol{h}(\mathbf{1}), \boldsymbol{h}(2), \ldots \boldsymbol{h}(\boldsymbol{n})$ as shown in figure 5 , are picked up from the $\boldsymbol{T F P \boldsymbol { x }}$ around the peak point, and are approximated by the Gaussian curve $\boldsymbol{G}(\boldsymbol{i})$. The tip rate (T.R.) is then defined as 


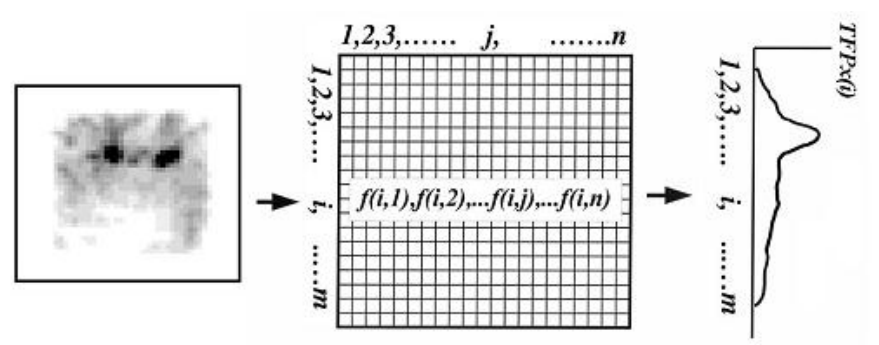

Fig.4 2-D representation of buttock pressure distribution and horizontal top-five projection.

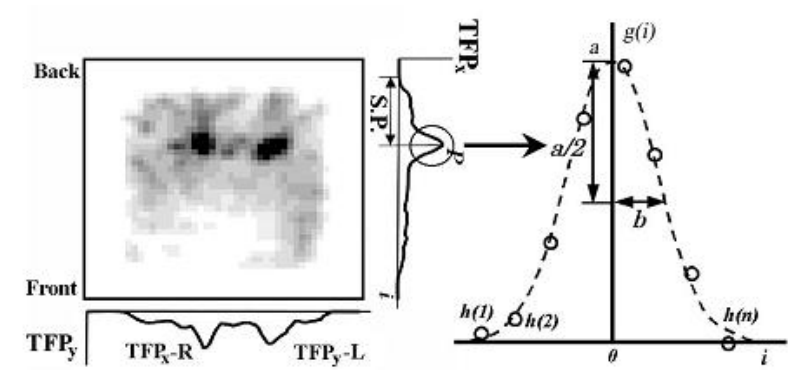

Fig.5 Horizontal top-five projection (TFPx), vertical top-five2 projection (TFPy) and an approximate Gaussian curve.

$$
T \cdot R .=\frac{a}{2 b}
$$

where $\boldsymbol{a}$ is the peak value of the approximated Gaussian curve, and $\boldsymbol{b}$ is defined as

$$
G(b)=\frac{a}{2}, G(0)=a \text {. }
$$

We define the length between the maximum point of the TFPx and the back end of the buttock as the sitting position (S.P.). The sitting balance (S.B.) is defined by

$$
\text { S.B. }=\frac{\left|T F P_{y-R}-T F P_{y-L}\right|}{T F P_{y-R}+T F P_{y-L}} \times 100
$$

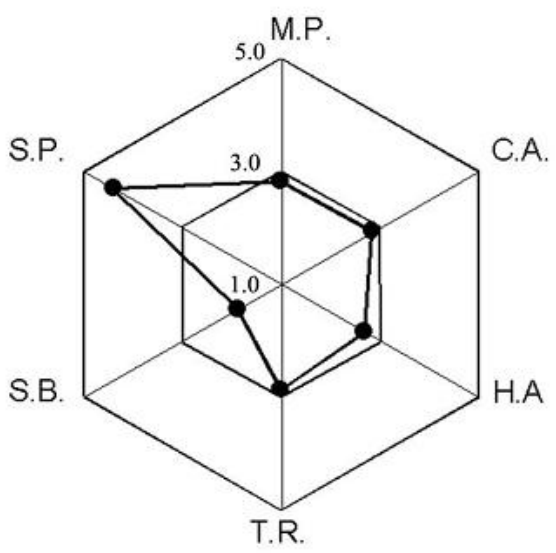

Fig.6 Hexagonal representation of 6 parameters. where $\boldsymbol{T F P} \boldsymbol{H}-\boldsymbol{R}$ is the value of the maximum right point in the $\boldsymbol{T F P y}$, and $T F P y-L$ is the value of the maximum left point in the TFPy.

The six parameters are represented on the hexagonal radar plot shown in Figure 6. In this figure, an inner perfect hexagon shows the mean of each parameter for $25 \mathrm{SCI}$ patients using the air cushion. Each parameter is put on each axis after normalization with standard deviation of each parameter. The medical staff can then learn the position and the approximate size of a high-pressure area from the 2-D representation of the pressure distribution, and the exact size of a high-pressure area from the H.A. on the radar plot. The T.R. shows the concentration of pressure. When the SCI patient has a large T.R. and M.P., the medical staff must carefully adjust the air quantity in the air cushion. When the SCI patient has a large S.P. on the radar plot, she or he sits anteriorly on the wheelchair. When the SCI patient has a large S.B. on the radar plot, the medical staff must assist the SCI patient in maintaining a correct posture.

\subsection{AIR ADJUSTMENT OF AIR CUSHION}

Figure 7 shows the relation between the contact area and the air pressure in cells, and between the maximum pressure and the air pressure in cells with a healthy subject. The maximum pres sure and the air pressure have a proportional relationship, whereas the contact area and the air pressure have a hyperbolic relationship. In this figure, the patient's weight is distributed evenly and over the widest surface area when the quantity of air in each cell is small. Due to the bottoming caused by the small

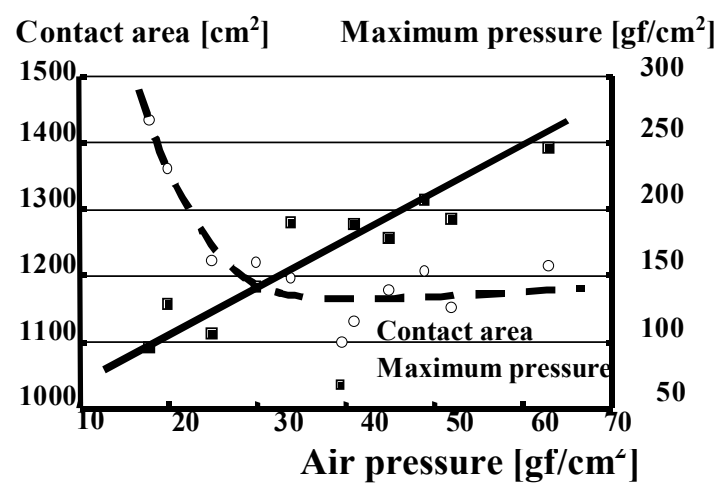

Fig. 7 Relation between contact area and air pressure, and between maximum pressure and air pressure. 


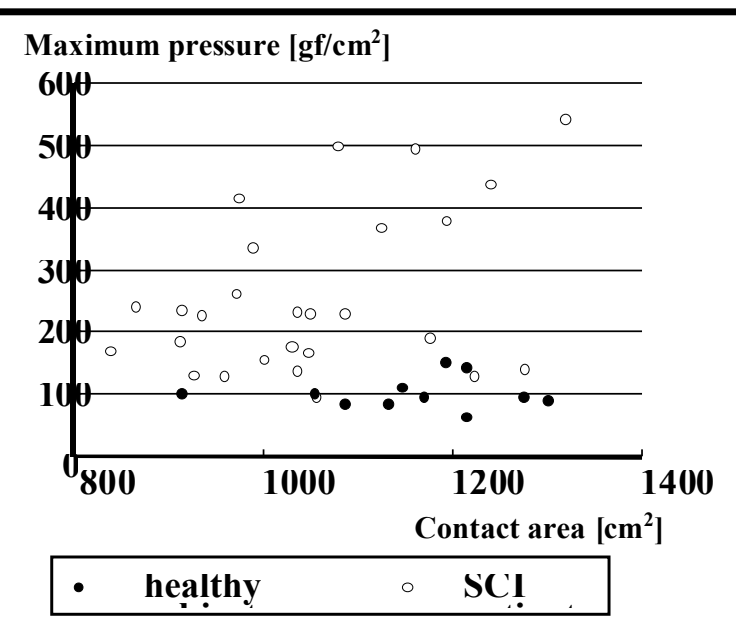

Fig.8 Contact area and maximum pressure of the air cushions adjusted to the atmosphere pressure.

quantity of air, however, the maximum pressure becomes large. Because the pressure relieving effect is large in the air cushion, it is important for medical staff to carefully adjust the air quantity in the cushion.

Figure 8 shows the relation between the maximum pressure and the contact area in 27 SCI patients and 12 healthy subjects after the adjustment to atmospheric pressure. The maximum pressure of the SCI patients ranged from 93.8 to $540.7 \mathrm{gf} /$ $\mathrm{cm}^{2}$; the average was $255.3 \mathrm{gf} / \mathrm{cm}^{2}$. The maximum pressure of the healthy subjects ranged from 81.8 to $151.9 \mathrm{gf} / \mathrm{cm}^{2}$, with an average of $101 \mathrm{gf} / \mathrm{cm}^{2}$. The maximum pressure of the SCI patients was much larger than the healthy subjects. The maximum pressure is greater among the SCI patients. The reason is thought to be the lack of muscle and fat around both ischial tuberosities, which occurs in many SCI patients after injury.

Because the maximum pressure differs among each SCI patient, and the influence of the air pressure on pressure distribution is large in the air cushion, it is important for medical staff to carefully adjust the air in the air cushion for each SCI patient. We measured the buttock pressure distribution of the SCI patient when he or she sat on an air cushion adjusted to the atmospheric pressure. We then evaluated the buttock pressure distribution of the SCI patient. Figure 9 shows the 2-D representation of the pressure distribution, the diagram of the relation between the body weight and air pressure, the diagram of the relation between the contact area and the maximum pressure, and the hexagonal radar plot. In this SCI patient, the high-pressure area was observed around both I.T. (ischial tuberosities), and in the S.C. (sacral coccygeal regions). Therfore, the medical staff can learn, from this representation, the position and the approximate size of a high-pressure area. Moreover, he or she can understand whether the SCI patient anteriorly or posterioly sits on their wheelchair. From the scatter diagram of the air pressure and the body weight, the medical staff find out whether the best air pressure adjustment has been made. From the radar plot and the scatter diagram of the maximum

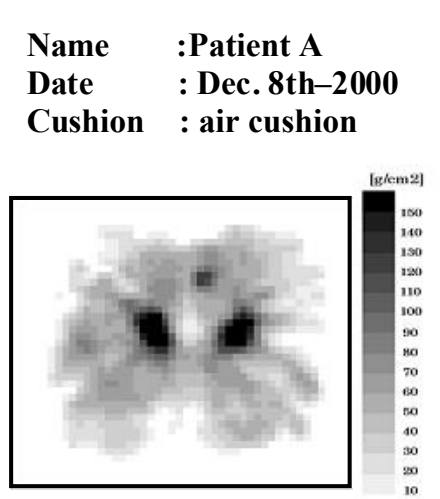

a) 2-D pressure distribution

Air pressure : $28[\mathrm{mmHg}]$ Contact area : $1087\left[\mathrm{~cm}^{2}\right]$ Right I.T. : $218\left[\mathrm{gf} / \mathrm{cm}^{2}\right]$ Left I.T. : $227\left[\mathrm{gf} / \mathrm{cm}^{2}\right]$ S.C. Region : $119\left[\mathrm{gf} / \mathrm{cm}^{2}\right]$

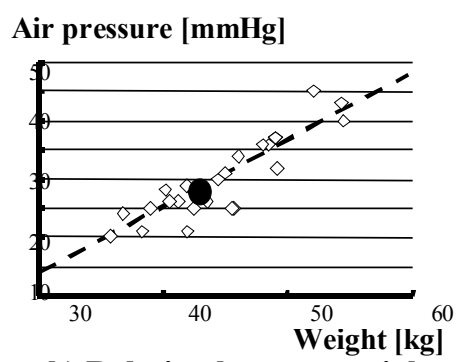

b) Relation between weight and air pressure

Maximum pressure $\left[\mathrm{gf} / \mathrm{cm}^{2}\right]$

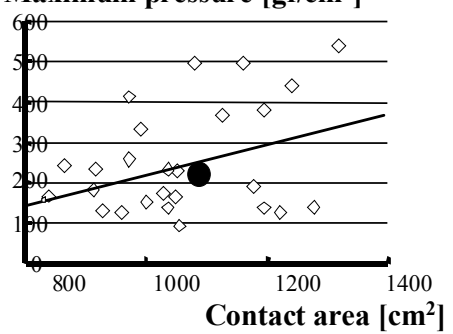

c) Relation between contact area and maximum pressure

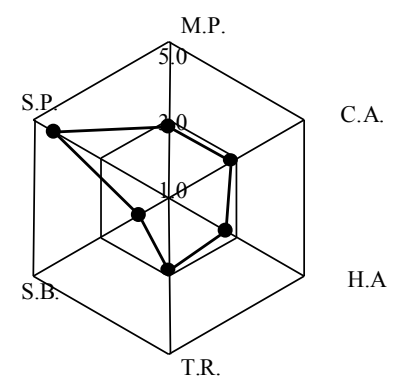

d) Radar chart

\section{Five Scale E'valuation}

$\begin{array}{ll}\text { Maximum pressure(M.P.) } & 2.8 \text { (Normal) } \\ \text { Contact area(C.A.) } & 2.8 \text { (Normal) } \\ \text { High pressure area(H.A.) } & 2.7 \text { (Normal) } \\ \text { Tip rate (T.R.) } & 2.9 \text { (Normal) } \\ \text { Sitting balance (S.B.) } & 1.9 \text { (Good) } \\ \text { Sitting position (S.P.) } & 4.4 \text { (Shallow) }\end{array}$

Fig. 9 The evaluating system of the buttock pressure distribution for prevention of pressure sores. 
pressure and the contact area, he or she can easily evaluate the pressure distribution, air adjustment, and posture of the patient on the wheelchair. When the M.P., T.R. and H.A. are large, the medical staff must carefully adjust the air quantity of the air cushion. The medical staff should instruct SCI patients on correct posture on the wheelchair, when his or her S.P. and S.B. are large.

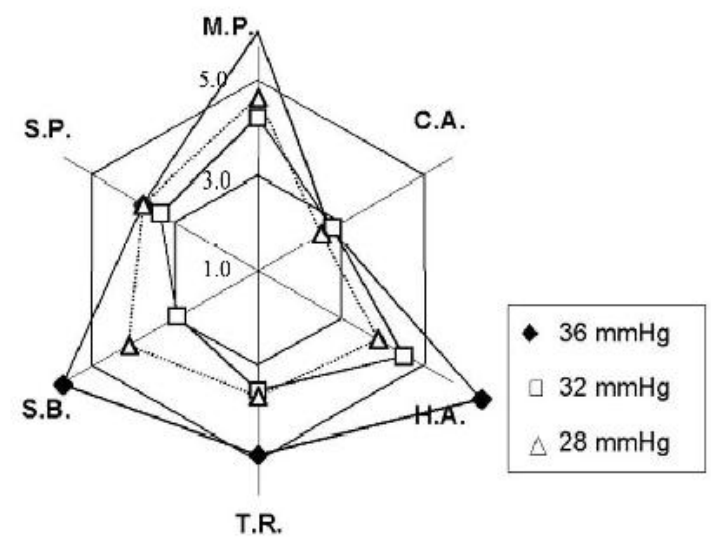

Fig.10 Hexagonal radar plot in adjustment of air cushion.

Figure 10 shows the results of the adjustment of the air cushion. Because the subject had a large maximum pressure, it was necessary for the medical staff to adjust the air pressure of the cushion. The air pressure was $36 \mathrm{mmHg}$ when the SCI patient sat on the air cushion that was adjusted to the atmospheric pressure. Because the M.P. was very large, the physician reduced the air pressure to $32 \mathrm{mmHg}$. The hexagonal radar plot became smaller than that of the air cushion with the large air press ure $(36 \mathrm{mmHg})$. Next, the physician let out a little more air from the air cushion, and the air pressure decreased to $28 \mathrm{mmHg}$. The hexagonal radar plot became larger than that of the air cushion with the middle air pressure $(32 \mathrm{mmHg})$. An air cushion with $32 \mathrm{mmHg}$ air pressure was thought to be suitable for this SCI patient.

\section{MEASUREMENT ON BED}

\subsection{PRESSURE RELIEVING EFFECT OF MATTRESS}

Many SCI patients use a pressure-relieving mattress to distribute their body weight evenly, in order to help prevent pressure sores. Quadriplegic patients have an especially high risk of developing pressure sores, because they sit or lie in bed for long periods of time. They often have meals, read books, and use the computer while sitting up in bed, as shown in Figure 11. Therefore, it is vital for medical staff treating these patients to measure the amount of buttock pressure on the bed, when the patient is using a pressure-relieving mattress.

Many pressure-relieving mattresses have been developed to reduce the interface pressure at particularly susceptible areas (the ischial tuberosities, sacro-coccygeal region and heels) on the bed. Figure 12 shows the two-dimensional pressure distributions of a healthy subject sitting on a wheelchair equipped with a urethane foam wheelchair cushion, and lying and sitting on a bed using the mattress. A high-pressure area is observed at both ischial tuberosities when the subject was sitting on the wheelchair cushion. In constant, the pressure was large at the sacro-coccygeal region when the subject sat or lay on the bed using the mattress.

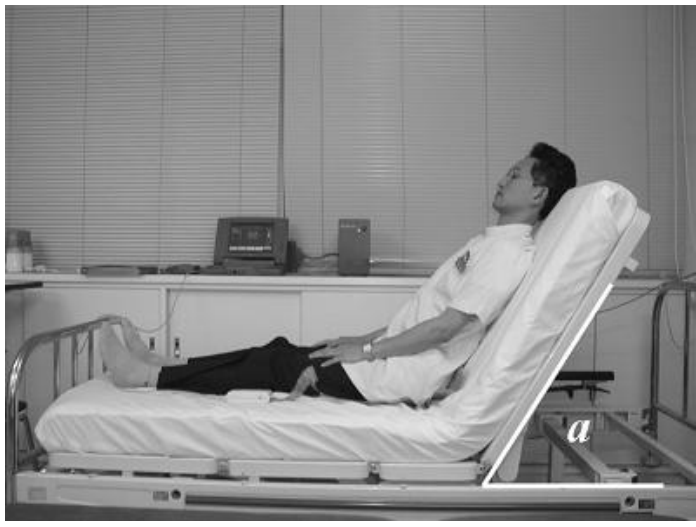

Fig.11 Sitting posture on the Gatch bed.

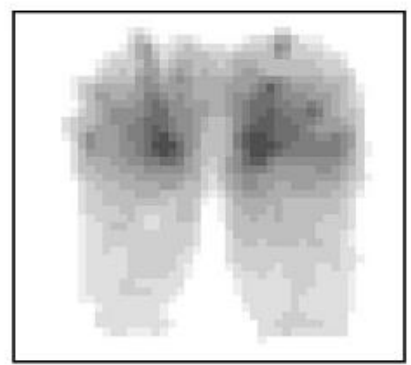

a) Wheel chair cushion (urethane foam)

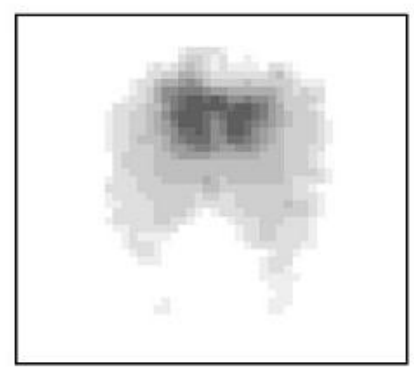

b) Mattress (polyester) Lying position
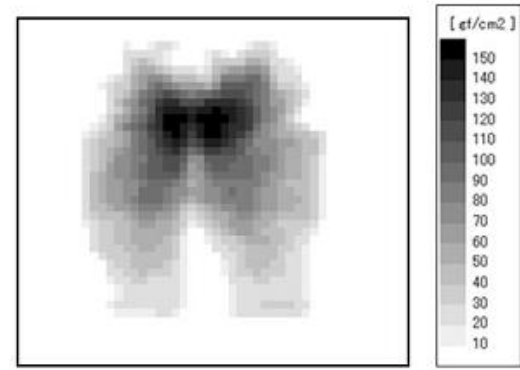

c) Mattress (polyester) Sitting position

Fig.12 Buttock pressure distribution on wheelchir and bed. 


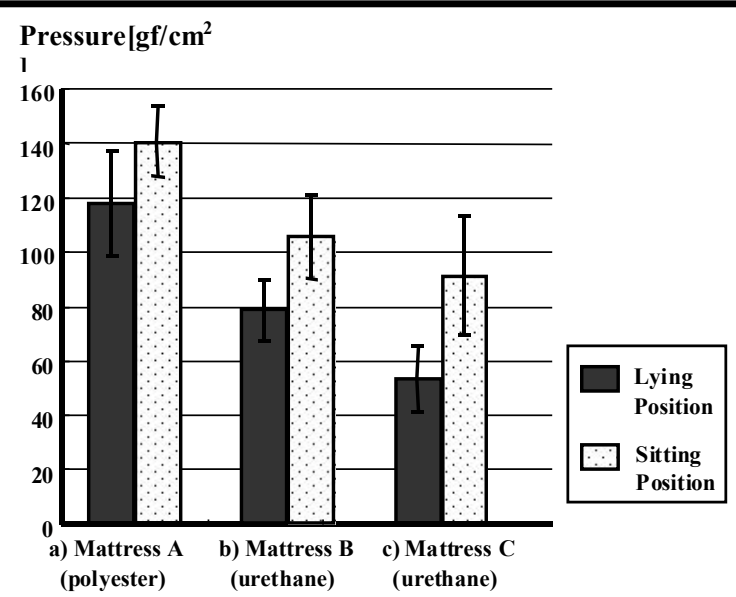

Fig.13 Maximum pressure at sacro-coccygeal region.

This figure shows that SCI patients, who spend a long time in bed, should be careful about the condition of their skin at their sacro-coccygeal region, because the pressure at the sacro-coccygeal region is great, not only when the patient is sitting, but also when he or she is spine position.

High level SCI patients, who cannot move their body by themselves, usually use an air mattress, because the prevention of pressure sores is the very important. But some SCI patients with mobility, never use mattresses made of fluid material such as air and water, because it is difficult for them to move, lift up, and transfer their body from bed to wheelchair on these kinds of mattresses. It is necessary for SCI patients and their medical staff to select the best mattress for each patient, taking into consideration the patient's pressure distribution and mobility on their bed. We measured the buttock pressure distribution of 14 healthy subjects using three types of mattress. SCI patients were able to move easily on Mattress A, which was made of polyester material, and many patients in our rehabilitation center use this mattress. SCI patients were able to move and push themselves up on Mattress $\mathrm{B}$, which was a polyurethane foam mattress. Mat-

Pressure $\left[\mathrm{gf} / \mathrm{cm}^{2}\right]$

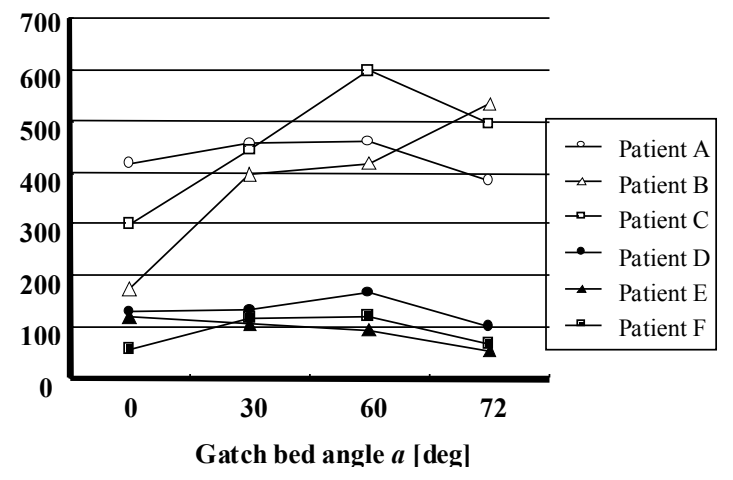

Fig. 14 Pressure at the sacro-coccygeal region. tress $\mathrm{C}$ was made of soft polyurethane foam, and the SCI patients who had a good ability to push themselves up were able to move and push them up on this mattress. Figure 13 shows the maximum pressure at the sacro-coccygeal region in the sitting and spine position for 14 healthy subjects. The pressure exerted in the sitting position was over $20 \%$ greater than that in the spine position. The maximum pressure of mattress $B$ was about $20 \%$ smaller than mattress $\mathrm{A}$, and the maximum pressure of mattress $\mathrm{C}$ was over $30 \%$ smaller than mattress $\mathrm{A}$. We are able to reduce the pressure on the sacro-coccygeal region in the SCI patients to less than $80 \%$ using the soft urethane foam mattress. Significant differences were observed between mattress A and mattress B, mattress B and mattress $C$, and mattress $A$ and mattress $C$ in the spine position using the Student's t-test $(\mathrm{P}<0.01)$. Significant differences were observed between mattress $\mathrm{A}$ and mattress $\mathrm{B}$, and mattress $\mathrm{A}$ and mattress $C(P<0.01)$. No significant difference was

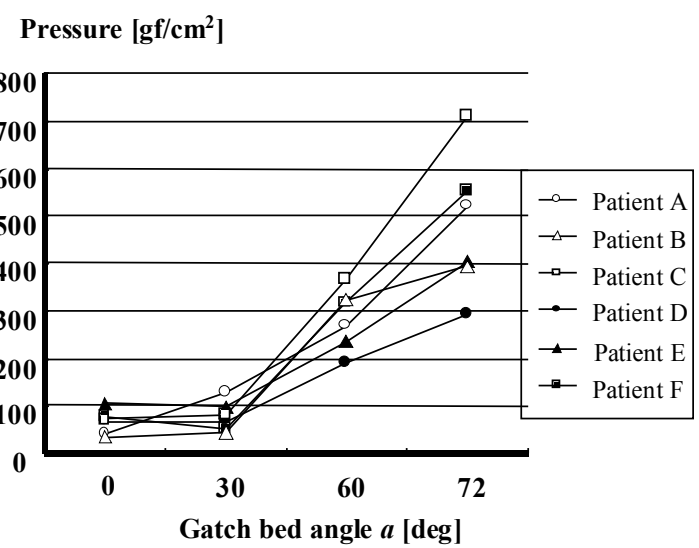

Fig. 15 Pressure at the ischial tuberosity .

found between mattress $\mathrm{B}$ and mattress $\mathrm{C}$ in the sitting position $(\mathrm{P}<0.01)$.

Many quadriplegic patients spend most of the day on a Gatch bed. They sometimes sit and elevate the head of the bed, as shown in Figure 11. It is very important for physicians and nurses to evaluate the pressure distribution of the mattress, and the patient's posture on the bed. Figure 14 shows the pressure at the sacro-coccygeal region of six SCI patients. The Gatch bed angle $\boldsymbol{a}$ equals 0,30 , 60 and 72 [deg]). Patients A, B, and C used mattress A made of polyester, and patients $\mathrm{D}, \mathrm{E}$, and $\mathrm{F}$ used the air mattress. The three SCI patients' pressures at the sacro-coccygeal region were much higher than the healthy subjects' pressures in the spine position (Gatch bed angle equals $0[\mathrm{deg}]$ ) using mattress A, shown in Figure 13 and 14. When the Gatch bed angle was more than 30 [deg], the pressure of the SCI patients who were using mat- 
tress A were very high; more than $400\left[\mathrm{gf} / \mathrm{cm}^{2}\right]$. The pressure at the sacro-coccygeal region of the SCI patients who used the air mattress were small inall of the Gatch bed angles measured. The graph shows that the air mattress has a better pressure-relieving effect at the sacro-coccygeal region in all of the postures assumed by the patients' than mattress A.

The pressure at the ischial tuberosity on the spine position (Gatch bed angle $\boldsymbol{a}$ was 0 [deg]) and the Gatch bed angle setting 30 [deg] was less than 150 $\left[\mathrm{gf} / \mathrm{cm}^{2}\right]$, as shown in Figure 15. The pressure at the ischial tuberosity was more than $200\left[\mathrm{gf} / \mathrm{cm}^{2}\right]$ on a Gatch bed angle setting of 60 [deg]. The pressure was very high (more than $300\left[\mathrm{gf} / \mathrm{cm}^{2}\right]$ ) on the Gatch bed angle setting at the maximum degree of $(72[\mathrm{deg}])$. According to this figure, it is clear that the air mattress and the polyester mattress have no pressure relieving effect at the ischial tuberosities when the patient is sitting.

Figures 14 and 15 show that the air mattress has the greatest pressure relieving effect at the sacrococcygeal region in all postures assumed on the bed. However, the mattress has no pressure relieving effect at the ischial tuberosities when the patient is sitting up in bed. SCI patients who are at high risk for developing pressure sores should use an air mattress or a mattress, which has a pressure-relieving effect, and should not sit up in bed for a long time.

\section{CONCLUSION}

In rehabilitation medicine, it is important to reduce the SCI patients' buttock pressure when sitting in a wheelchair and in bed, in order to reduce the occurrence of pressure sores.

In this study, we proposed a supporting system for making air adjustments of the air cushion. Using this system, we were able to evaluate the buttock pressure distribution for each SCI patient, and decide on a suitable air pressure for the air cushion used by each SCI patient.

We measured the buttock pressure on the bed using three types of mattresses to evaluate the pressure relieving effect of the mattress. The results show that SCI patients should take care not to put too much pressure on the skin at the sacro-coccygeal region, especially when they spend a long time sitting up in bed. The best pressure relieving effect was observed in the polyurethane foam mattress, because the maximum pressure of the polyurethane mattress was $20 \%$ less than that of the polyester mattress. We measured the buttock pressure in four kinds of postures on the Gatch bed, in order to indicate the differences of pressure distribution in sitting postures on the bed. The pressure at the ischial tuberosities rose the Gatch bed angle increased. The air mat- tress has a high pressure relieving effect at the sacrococcygeal region in all postures on the bed. However, SCIpatients should not sit up in bed for a long time, because the air mattress and the polyester mattress had no pressure relieving effect on the ischial tuberosities when the patient was sitting. In the future, measurement of buttock pressure in patients has to be made using many kinds of mattresses, for the purpose of both suggesting a suitable mattress for each SCI patient, and giving each patient instructions on posture when in bed.

\section{REFERENCES}

[1] A. Tokuhiro, "A study of the incidence of the pressure sore in rehabilitated paraplegic patient," Jap. J. Trauma. Occupy. Med., 38, pp.538-541, 1990

[2] K. Fujiie, “A questionnaire on pressure sore with spinal injuries," Rehabilitation Engineering R\&D S.C.I., pp. 123-133, 1992.

[3] T. Inoue, A. Nakajima, T. Aoyama,"The pressure relieving effects of the wheelchair cushion in Royal Perth Rehabilitation Hospital," Jap. J. Trauma. Occupy. Med., 38, pp. 582-587, 1990.

[4] Brienza DM, Karg PE and Brubaker CE , "Seat cushion design for elderly wheelchair users based on minimization of soft tissue deformation using stiffness and pressure measurements, " IEEE Transactions of Rehabilitation Engineering,4(4), pp. 320-327, 1996.

[5] Conner LM and Clack JW, "In vivo (CT scan) comparison of vertical shear inhuman tissue caused by various support surfaces," Decubitus, vol.6, No.20, pp. 20-23, pp. 26-28, 1993.

[6] R. Palmieri, G.T. Haelen, G.V.B. Cochran "A comparison of sitting pressure on wheelchair cushions as measured by air cell transducers and miniature electronic transducers, "Bulletinn of prosthetics research, 17, pp. 5-8, 1980.

[7] T. K. K. Koo, F. T. Mark, Y. L. Lee K "Posture effect on seating interface biomechanics: comparison between two seating cushions," Arch. Phys. Med. Rehabil., 77, pp. 40-47, 1996.

[8] Y. Tanimoto, H. Takechi, H. Nagahata, H. Yamamoto "The study of pressure distribution in sitting position on cushions for patient with SCI (spinal cord injury)," IEEE Transaction on instrumentation and measurement, 47(5), pp. 1239-1243,Octorber, 1998

[9] Y. Tanimoto, H. Takechi, H. Nagahata, H. Yamamoto, "Pressure measurement of air cushion for patient," IEEE Transaction on instrumentation and measurement, 49(3), pp. 666670, June, 2000 


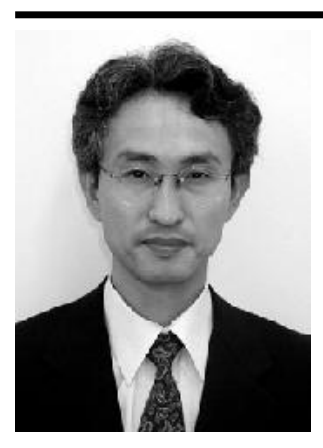

Yoshio Tanimoto was born in 1961. He received the B.S. and M.S. degrees in electrical engineering from Okayama University, Okayama, Japan in 1984 and 1986, respectively, and the Ph.D. degree in medical engineering from Hyogo University of Teacher Education, Katogun-Yashirocho, Hyogo, Japan, in 1999.

In 1988, he joined the Kibikogen Rehabilitation Center for Employment Injuries, JobogunKayocho, Okayama, Japan, where he is a Rehabilitation Engineer. His current interest is in the area of rehabilitation engineering.

Hirosuke Takechi was born was born in 1962. He received the degree of Medical Science from Okayama University, Okayama, Japan, in 1999.

In 2001, he joined the Kibikogen Rehabilitation Center for Employment Injuries, Jobogun-Kayocho, Okayama, Japan, where he is an ortho-

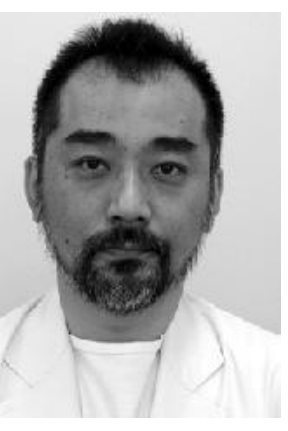
pedic surgeon. His research interest is the area of biomechanics.

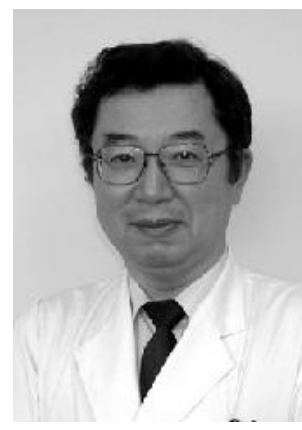

Akihiro Tokuhiro was born in 1950. He received the degree of Medical Science from Okayama University, Okayama, Japan, in 1986.

In 1987, he joined the Kibikogen Rehabilitation Center for Employment Injuries, Jobogun-Kayocho, Okayama, Japan, and he has been working for this center in the rehabilitation medicine, as a specialist in the medical rehabilitation of spinal cord injury.
Hideo Takechi was born in 1930. He received the degree of Medical Science from Okayama University, Okayama, Japan, in 1959.

In 1965, he was appointed Lecturer and in 1979 he became Associate Professor of Orthopedic Surgery at Okayama University Hospital, Okayama, Japan. In 1965-66 he worked at Heidelberg University, Heidelberg, Germany, as the Fellow of Alexander von Hunboldt Foundation. In 1987 he became Director of Kibikogen Rehabilitation Center for Employment Injuries, Jobogun-Kayocho, Okayama, Japan. His current interests are in biomechanics and prosthetics, and orthotics.

Dr. Takechi is a member of the Sociŭtŭ Internationale de Chirurgie Orthopŭdique et de Traumatologique (SICOT) and the International Society for Prosthetics and Orthotics (ISPO).

Hideki Yamamoto was born in 1944. He received the B.S. degree in electrical engineering from Okayama University, Okayama, Japan, in 1966, and the Ph.D. degree in electrical engineering from Kyoto University, Kyoto, Japan, in 1983.

He is currently a Professor in the Department of Educa-

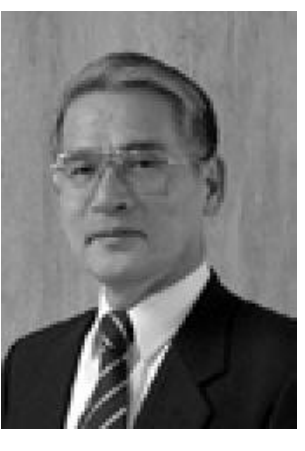
tion at Okayama University. His research interests include image processing and signal processing. 